\title{
A Low-Complexity Decision-Feedback Equalization Based on Noise-Prediction
}

\author{
Xuejiao Li $i^{\mathrm{a}, *}$, Songyi Xu \\ The 54th Research Institute of China Electronics Technology Group Corporation, Shijiazhuang 050081, \\ China. \\ alixuejiao06@qq.com
}

Keywords: troposcatter communication, noise-prediction, decision-feedback equalization, low-complexity

\begin{abstract}
It is necessary to equalize the received signals to weaken serious multipath effects in troposcatter communication systems. According to the multipath channel model in scatter communication systems mentioned in related papers, a low-complexity decision-feedback equalization based on noise-prediction is proposed by partial coefficients of curve fitting prediction in nonlinear decision feedback equalizer when time delay power spectrum is under the condition of exponential decay. Simulation results showed that the method proposed in this paper has a lower complexity as well as good BER performance.
\end{abstract}

\section{Introduction}

Troposcatter communication depends on a typical BLOS (Beyond Line of Sight) channel, it transmits radio signals by forward scattering through troposphere scatter, it is characterized by large capacity, long range, high confidentiality, etc. However, long distance transmission in serious troposcatter channels usually causes obvious multipath fading and strong time-varying, normally, it is difficult to estimate the distribution and time delay in real multipath channels. Therefore, in this paper, we take Rayleigh channel [1] as simulation model in troposcatter communication systems.

It is necessary to equalize the received signals with severe inner-symbol interference caused by obvious multipath fading effects in troposcatter channel. At present, it has been discussed and researched extensively using linear frequency-domain equalizations based on zero-forcing (ZF) and minimum mean square error (MMSE) method [2-4]. Furthermore, a nonlinear decision-feedback equalization fully using the output of decoder in both time and frequency domain is proposed [5]. However, this decision-feedback equalization will cause error propagation. In Ref. [6,7], decision-feedback equalization based on noise-prediction (DFE-NP) is used to equalize signals by predicting noise which can weaken error propagation and get better performance, but it has a high complexity.

In this paper, we proposed a low-complexity decision-feedback equalization based on noise-prediction, compared with ZF, MMSE and DFE-NP presented in in iteration 1 and 2, the simulation results showed that the method we proposed has lower complexity and a similarly good performance.

\section{System Model}

We consider a single-input single-output (SISO) and single-carrier system is transmitted by blocks with cycle prefix (CP) and there are $\mathrm{N}$ information symbols in every block, which can be expressed by $\boldsymbol{x}_{\boldsymbol{n}}, n=0,1, \cdots, N-1$ and its average energy is $\delta_{x}^{2}$. The channels have a time-invariant impulse response during each block and may be varying in another block transmission period. In addition, we assume that the channels remain unchanged with its longest 
multipath delay being L symbol periods. And the last $N_{c p}$ symbols of each block, as cyclic prefix, would be inserted in front of that block to remove the inter-block interference. When $N_{c p}$ is no less than L, the received signals of the block excluding the part of CP can be expressed by

$$
\boldsymbol{y}=\boldsymbol{h} \boldsymbol{x}+\boldsymbol{w}
$$

where $\boldsymbol{y}=\left[y_{0} y_{1} \cdots y_{N-1}\right]^{\mathrm{T}}, \boldsymbol{x}=\left[x_{0} x_{1} \cdots x_{N-1}\right]^{\mathrm{T}}, \boldsymbol{w}=\left[w_{0} w_{1} \cdots w_{N-1}\right]^{\mathrm{T}} \cdot \boldsymbol{w}$ denotes the vector of the additive white Gaussian noises with the variance $\delta_{w}^{2}$. $\boldsymbol{h}$ is a $N \times N$ cycle matrix which first column is $\boldsymbol{h}_{0}=\left[\mathrm{h}_{0} \mathrm{~h}_{1} \cdots \mathrm{h}_{\mathrm{L}-1} 0 \cdots 0\right]^{\mathrm{T}}$ and $h_{n}$ is the channels' impulse responses. Cycle matrix can be diagonalizable, so $\boldsymbol{h}=\boldsymbol{F}^{\mathrm{H}} \boldsymbol{H F}, \boldsymbol{H}$ is a diagonal matrix. $\boldsymbol{F}$ denotes Fourier transform matrix and $\boldsymbol{F}^{\mathrm{H}}$ denotes inverse Fourier transform matrix. The elements of $\boldsymbol{H}$ are

$$
H_{k}=\sum_{0}^{N-1} h_{n} \exp (-\mathrm{j} 2 \pi k n / N), k=0,1 \cdots N-1
$$

and expressed in the frequency domain

$$
\boldsymbol{Y}=\boldsymbol{H} \boldsymbol{X}+\boldsymbol{W}
$$

\section{Low-complexity decision-feedback equalization based on noise-prediction}

\subsection{ZF, MMSE and DFE-NP}

ZF and MMSE in frequency domain are often-used linear equalizations who have simple structure but worse performance. Nonlinear equalization had been researched a lot for its better property. Now we would like to talk about decision-feedback equalization based on noise-prediction.

The structure of single-carrier receiver using DFE-NP is shown as Fig. 1, it contains a linear equalizer in frequency domain and a noise predictor in time domain.

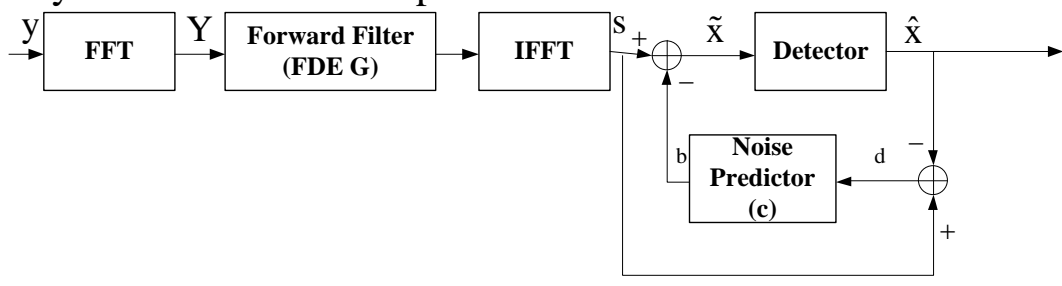

Fig. 1 structure of single-carrier receiver using DFE-NP

It is assumed that receiver has ideal channel estimation and synchronization, the receive signals, after feed forward filter equalization and Fourier inverse transformation, can be expressed

$$
\boldsymbol{s}=\boldsymbol{F}^{\mathrm{H}}(\boldsymbol{G} \boldsymbol{Y})=\boldsymbol{F}^{\mathrm{H}}(\boldsymbol{G H} \boldsymbol{X}+\boldsymbol{G W})
$$

where $\boldsymbol{s}=\left[s_{0}, s_{1}, \cdots, s_{N-1}\right]^{\mathrm{T}}, \boldsymbol{G}=\left[G_{0}, G_{1}, \cdots, G_{N-1}\right]^{\mathrm{T}} . \boldsymbol{G}$, a cycle diagonal matrix, is made by coefficients of DFE. $\boldsymbol{d}=\boldsymbol{s}-\hat{\boldsymbol{x}}$ is the input of noise predictor. $\hat{\boldsymbol{x}}$ is the symbols after hard decision. The input of detector is

$$
\tilde{\boldsymbol{x}}=\boldsymbol{s}-(\boldsymbol{I}-\boldsymbol{c}) \boldsymbol{d}=\boldsymbol{s}-(\boldsymbol{I}-\boldsymbol{c})(\boldsymbol{s}-\hat{\boldsymbol{x}})
$$

where $\boldsymbol{c}$ is a $N \times N$ cycle matrix made by coefficients of noise predictor whose first column is $\left[1, c_{1}, c_{2} \cdots c_{B}, 0 \cdots 0\right]^{\mathrm{T}}$. No detective error is assumed, $\hat{\boldsymbol{x}}=\boldsymbol{x}$, so

$\boldsymbol{e}=\tilde{\boldsymbol{x}}-\boldsymbol{x}=\boldsymbol{c}(\boldsymbol{s}-\boldsymbol{x})$, using MMSE

$$
\begin{aligned}
J & =\mathrm{E}\left(\boldsymbol{e} e^{\mathrm{H}}\right)=\mathrm{E}\left[\boldsymbol{c}(\boldsymbol{s}-\boldsymbol{x})(\boldsymbol{s}-\boldsymbol{x})^{\mathrm{H}} \boldsymbol{c}^{\mathrm{H}}\right] \\
& =\boldsymbol{c}\left(\boldsymbol{F}^{\mathrm{H}} \boldsymbol{G}\left(\delta_{x}^{2} \boldsymbol{H} \boldsymbol{H}^{\mathrm{H}}+\delta_{w}^{2} \boldsymbol{I}\right) \boldsymbol{G}^{\mathrm{H}} \boldsymbol{F}-\delta_{x}^{2} \boldsymbol{F}^{\mathrm{H}} \boldsymbol{G} \boldsymbol{H} \boldsymbol{F}-\delta_{x}^{2} \boldsymbol{F}^{\mathrm{H}} \boldsymbol{H}^{\mathrm{H}} \boldsymbol{W}^{\mathrm{H}} \boldsymbol{F}+\delta_{x}^{2} \boldsymbol{I}\right) \boldsymbol{c}^{\mathrm{H}}
\end{aligned}
$$

$\delta_{x}^{2} \boldsymbol{H} \boldsymbol{H}^{\mathrm{H}}+\delta_{w}^{2} \boldsymbol{I}$ is considered to be a diagonal matrix, so 


$$
J=\operatorname{tr}\left(\mathrm{E}\left(\boldsymbol{e} \boldsymbol{e}^{\mathrm{H}}\right)\right)=\frac{\delta_{x}^{2}}{N} \sum_{l=0}^{N-1} \sum_{k=0}^{N-1}\left|\mathrm{e}^{\mathrm{j} \frac{2 \pi}{N} k l}\left(\sum_{m=0}^{B} c_{m} \mathrm{e}^{-\mathrm{j} \frac{2 \pi}{N} m k}\right)\right|^{2} \frac{\delta_{w}^{2}}{\delta_{x}^{2}\left|H_{k}\right|^{2}+\delta_{w}^{2}}, c_{0}=1
$$

then take derivative of $\boldsymbol{G}$ and $\boldsymbol{C}_{m}$, make them equal to 0 . Finally, we have

$$
\begin{gathered}
\boldsymbol{G}=\frac{\delta_{x}^{2} \boldsymbol{H}^{\mathrm{H}}}{\delta_{x}^{2} \boldsymbol{H} \boldsymbol{H}^{\mathrm{H}}+\delta_{w}^{2} \boldsymbol{I}} \\
\sum_{m=1}^{B} \sum_{k=0}^{N-1} c_{m} \mathrm{e}^{\mathrm{j} \frac{2 \pi}{N} k(n-m)} \frac{\delta_{w}^{2}}{\delta_{x}^{2}\left|H_{k}\right|^{2}+\delta_{w}^{2}}=-\sum_{k=0}^{N-1} \mathrm{e}^{\mathrm{j} \frac{2 \pi}{N} k n} \frac{\delta_{w}^{2}}{\delta_{x}^{2}\left|H_{k}\right|^{2}+\delta_{w}^{2}}, n=1 \cdots B
\end{gathered}
$$

We will get the coefficients of feedback equalizer through $B$ equations.

We can find that $\boldsymbol{G}$ is independent with $\boldsymbol{c}$ which means DFE-NP has a better flexibility and can be changed just by changing the tap number in feedback equalizer. In addition, matrix inversion should be figured out to get $\boldsymbol{c}$ from (9) so that the complexity would rise as exponent when B increases. Due to the matrix needed inverse is a Toepliz, the amount of calculation of inversion is $B^{2}+(B+1) \times N$. In fact, the bad multipath effect can affect tens and even hundreds of symbols which calls for enough taps. As a result, the complexity of matrix inversion is very large and the transmission speed and performance in troposcatter communication will be directly degraded.

\subsection{Low-complexity decision-feedback equalization}

Referring to DFE-NP and equation (9), $c_{m}$ is smaller and smaller when $m$ rises and the last a few part of $c_{m}$ are very small and changed little. This part of coefficients causes large complexity while has limited equalization effect. So we proposed coefficients prediction instead of calculation to reduce complexity.

First, we set a threshold and increase tap number $E$ from 1 to $B$. Then calculate $c_{E}$ for every $E$ and judge if there are coefficients under or equal to the threshold. If $c_{i}$ is under or equals to the threshold for the first time when $E=i$, we predict the last $(B-i)$ coefficients using the first $i$ coefficients and don't calculate any more.

We assume that the coefficients suit that (we ignore the imaginary part for convenient)

$$
f=a \mathrm{e}^{-b x}
$$

where $f$ is dependent variable and $x$ is independent variable. We need to calculate $a$ and $b$ to minimum

$$
\gamma^{2}=\sum_{i=1}^{E}\left[c_{i}-a \mathrm{e}^{-b i}\right]^{2}, \quad(i=1 \cdots E)
$$

using E couples $\left(i, c_{i}\right)(i=1 \cdots E)$.

Because of the nonlinearity characteristic of exponent curve, the method of calculating the extreme value of multivariate function can't be obtained by linear least square to get the estimation value of the parameters. In order to reduce the calculation, we take the following method.

We incidentally select two from $E$ pairs of observed data and use them in $f=a \mathrm{e}^{-b x}$ to get $C_{E}^{2}$ pairs of $(a, b)$.

$$
\left\{\begin{array}{l}
a=\exp \left(\ln c_{n}+\frac{\ln c_{m}-\ln c_{n}}{n-m} n\right) \\
b=\frac{\ln c_{m}-\ln c_{n}}{n-m}
\end{array}, n 、 m=1 \cdots E\right.
$$

Average the $C_{E}^{2}$ pairs of $(a, b)$ and get $(\bar{a}, \bar{b})$. Calculate correlative $\gamma^{2}$ for every $(a, b)$ and 
compare their values. We take the smallest $\gamma^{2}$ and its $(a, b)$ as final parameters for the last $(B-i)$ decision-feedback coefficients' prediction. In this way, the amount of calculation is $\frac{3}{2} E^{3}+\frac{1}{2} E^{2}+(2+N) E+N$.

\section{Simulation Results}

According to the introduction of the above equalizations, we take the channel model introduced in [3]. Each path has independent Rayleigh fading and its time delay power spectrum decayed as exponent distribution. Considering the multipath time delay of troposcatter channel can be up to tens of symbols, we set the channel length to 64, the longest time delay to 20 symbols. The specific simulation parameters are shown in Table 1.

Table 1 Simulation Parameters

\begin{tabular}{|c|c|}
\hline Simulation Parameters & Values \\
\hline Length of Block & 576 \\
\hline FFT number & 512 \\
\hline CP length & 64 \\
\hline Modulation & QPSK \\
\hline the longest time delay(symbol) & 20 \\
\hline Decision-feedback Tap (B) & 18 \\
\hline
\end{tabular}

It is assumed that the system has ideal channel information and synchronization.

Firstly, the basic frequency domain equalizations-ZF, MMSE and DFE-NP were simulated. Their BER curves were shown in Fig. 2.

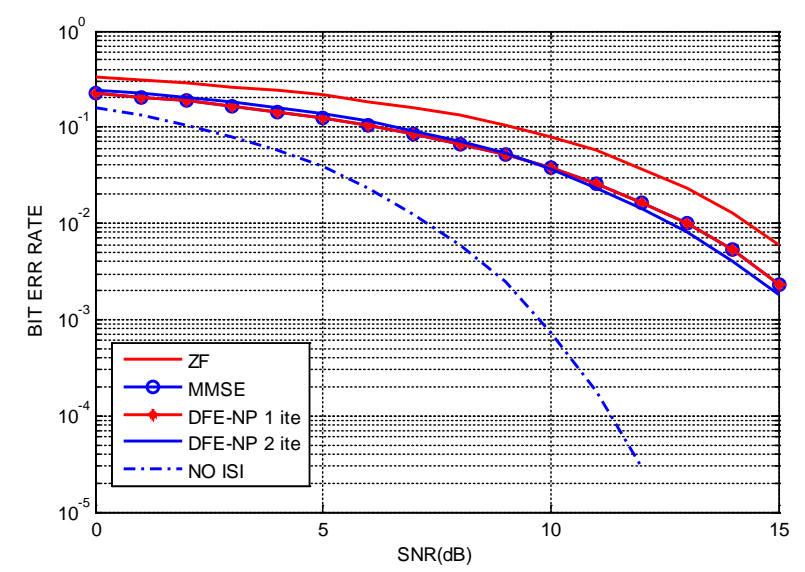

Fig. 2 Comparison among ZF, MMSE and DFE-NP

In the first iteration, there is no feedback information in DFE-NP so that its performance is same as that of MMSE. In the second iteration, the performance of DFE-NP is worse than that of MMSE when SNR is small due to the existence of error propagation and better than that when SNR is bigger. There also is a no-ISI curve in Fig. 2 for comparison.

Then, we simulated DFE-NP and the method we proposed (DFE-NP-S) in the same system when $\mathrm{B}$ is 18 and thresholds are $0.5,0.3$ and 0.15 . The amounts of calculation were shown in Table 2 . 
Table 2 Comparison of Complexity

\begin{tabular}{|c|c|c|c|}
\hline Algorithm & $\begin{array}{c}\text { Decision-feedback } \\
\text { Tap(B) }\end{array}$ & $\begin{array}{c}\text { Number of Accurate } \\
\text { calculation(E) }\end{array}$ & Complexity \\
\hline DFE-NP & 18 & 18 & 10052 \\
\hline DFE-NP-S(0.5) & 18 & 2(Relative to SNR(0 16)) & 1554 \\
\hline DFE-NP-S(0.2) & 18 & $\begin{array}{c}3 / 4(\text { Relative to } \\
\text { SNR(0 16) }\end{array}$ & $2672(E=4)$ \\
\hline DFE-NP-S(0.15) & 18 & $\begin{array}{c}\text { 5/6(Relative to } \\
\text { SNR(0 16) }\end{array}$ & $3938(E=6)$ \\
\hline
\end{tabular}

It can be found from Table 2 that the complexity of DFE-NP-S is reduced in the same condition with DFE-NP and the complexity changes when threshold changes. Fig.3 shows the comparison between DFE-NP and DFE-NP-S when B is 18 and thresholds are 0.5, 0.3 and 0.15.

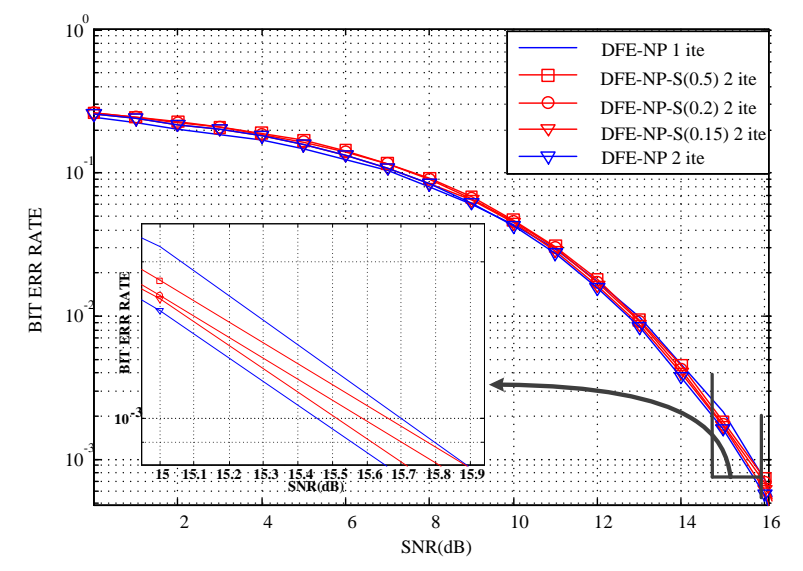

Fig.3 Comparison between DFE-NP and DFE-NP-S

We can see from Fig.3 that DFE-NP-S has very closely performance to DFE-NP while its complexity has reduced a lot when multipath channel's time delay power spectrum decayed as exponent distribution. When BER is $10^{-3}$, the loss of BER is smaller than $0.1 \mathrm{~dB}$ between DFE-NP-S and DFE-NP while threshold is 0.15.

\section{Conclusions}

In this paper, we proposed a low-complexity decision-feedback equalization based on noise-prediction, introduced its channel model, analyzed relative parameters and comparatively simulated system performances. It is verified that the method has pretty good BER performance while its complexity is lower and consequently it is suitable to be applied in the troposcatter communication system with severe multipath effect.

\section{References}

[1] Ohno S, Teo K A D, Performance of single-carrier block transmissions over multipath fading channels with MMSE equalization, Proceedings of the 2005 IEEE Global Telecommunications Conference (GLOBECOM’05). Missouri. 2005, pp.2129-2133.

[2] Pancaldi F, Vitetta G, Single-carrier Frequency domain equalization, IEEE Single Processing Magazine. 25 (2008) 37-56.

[3] Pancaldi F, Vitetta G M, Block channel equalization in the frequency domain, IEEE Transaction on Communication. 53 (2005) 463-471.

[4] XUE Zhenzhen, LI Li, Research on MIMO Single-Carrier Frequency Domain Equalization Algorithm in HF Communication, Radio Communications Technology. 41 (2015) 17-19, 36. 
[5] Falconer D, Ariyavisitakul S, Benyamin-Seeyar A, et al, Frequency domain equalization for single-carrier broadband wireless systems, IEEE Communications Magazine. 40 (2002) 58-66.

[6] Zhu Y, Letaief K B, Single carrier frequency domain equalization with time domain noise prediction for wideband wireless communications, IEEE Transaction on Wireless Communications. 5 (2006) 3548-3557.

[7] Zhu Y, Letaief K B. Single carrier frequency domain equalization with noise prediction for broadband wireless systems. In Proc. IEEE Global Telecommunications Conf. (GLOBECOM'04). Dallas. 2004, pp. 3098-3102. 